\title{
Spreading Characteristics and Thrust of Jets From Asymmetric Nozzles
}

K.B.M.Q. Zaman

Lewis Research Center

Cleveland, Ohio

Prepared for the

34th Aerospace Sciences Meeting and Exhibit

sponsored by the American Institute of Aeronautics and Astronautics Reno, Nevada, January 15-18, 1996

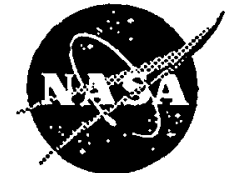

National Aeronautics and Space Administration

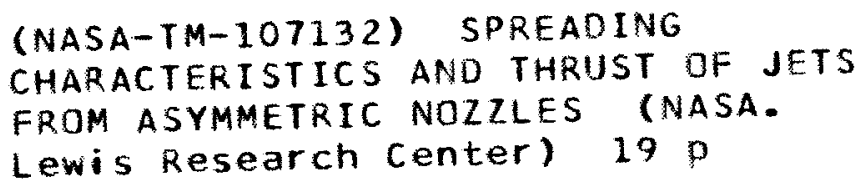




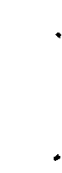




\title{
SPREADING CHARACTERISTICS AND THRUST OF JETS FROM ASYMMETRIC NOZZLES
}

\author{
by
}

\author{
K. B. M. Q. Zaman \\ NASA Lewis Research Center \\ Cleveland, OH 44135
}

\begin{abstract}
The spreading characteristics of jets from several asymmetric nozeles are studied in comparison to those of an axisymmetric jet, over the Mach number $\left(M_{j}\right)$ range of 0.3 to 1.96 . The effect of tabs in two cases, the axisymmetric nozwle fitted with four tabs and a rectangular nozzele fitted with two large tabs, is also included in the comparison. Compared to the axisymmetric jet, the asymmetric jets spread only slightly faster at subsonic conditions, while at supersonic conditions, when screech occurs, they spread much faster. Screech profoundly increases the spreading of all jets. The effect varies in the different stages of screech, and the corresponding unsteady flowfield characteristics are documented via phase-averaged measurement of the fluctuating total pressure. An organization and intensification of the aximuthal vortical structures under the screeching condition is believed to be responsible for the increased spreading. Curiously, the jet from a 'lobed mixer' nozzle spreads much less at supersonic conditions compared to all other cases. This is due to the absence of screech with this nozzle. Jet spreading for the two tab configurations, on the other hand, is significantly more than any of the notab cases. This is true in the subsonic regime, as well as in the supersonic regime in spite of the fact that screech is essentially eliminated by the tabs. The dynamics of the streamwise vortex pairs produced by the tabs cause the most efficient jet spreading thus far observed in the study.
\end{abstract}

\section{Nomenclature}

$\mathrm{C}_{\mathrm{f}} \quad$ Measured thrust normalized by ideal thrust. Ideal thrust is obtained from actual mass flux through nozzle, and assuming uniform, convergent flow.

D Equivalent diameter based on nozzle exit area.

$f_{s} \quad$ Screech frequency. m Mass flux obtained from Pitot probe survey at a given $\mathbf{x}$.

$m_{E} \quad$ Mass flux at nozzle exit obtained from $\mathrm{P}_{\mathrm{T}} / \mathrm{p}_{\mathrm{A}}$ assuming uniform flow.

M Mach number.

$M_{\text {MAX }}$ Maximum Mach number at a given X.

$M_{J} \quad$ Jet Mach number assuming fully expanded flow for a given $P_{T} / P_{A}$.

$\mathrm{p}_{\mathrm{A}} \quad$ Ambient pressure.

$\mathbf{P}_{\mathrm{T}} \quad$ Plenum chamber pressure.

St Strouhal number of screech, $\mathrm{f}_{S} \mathrm{D} / \mathrm{U}_{\mathrm{E}}$.

$T$ Jet thrust.

U Mean streamwise velocity.

$U_{E} \quad$ Jet velocity at nozzle exit.

$U_{M A X}$ Maximum $U$ at a given $x$.

$\mathbf{x}, \mathbf{y , z}$ Streamwise and transverse coordinates.

\section{Introduction}

Jets from asymmetric nozzles have been known to spread and mix faster than their axisymmetric counterparts. For example, Refs. 1 and 2 had shown that the overall spreading of small aspect ratio elliptic jets was clearly more than that of an axisymmetric jet. There were many studies on jets from rectangular nozzles, ${ }^{3.7}$ some of which also indicated more vigorous entrainment characteristics. While most of the studies cited above were for low Mach number subsonic jets, a similar inference was also made for supersonic elliptic jets. ${ }^{8}$ The possible impact of faster jet spreading on noise reduction was addressed in Ref. 9.

Based on the available data, however, a clear and direct comparison of the spreading characteristics of jets from the various nozzles has been difficult. Part of the difficulty is due to the fact that mixing and spreading of a jet can be defined in a number of ways, and different 
studies have used different parameters for that purpose. Many parameters, such as streamwise variation of the jet half-velocity-width or the centerline velocity, would only provide a partial description and could even be misleading. Another difficulty stems from differences in the operating conditions in the previous studies, especially in Mach number. It is well known that mixing layer spreading decreases with increasing compressiblity at higher Mach numbers. ${ }^{10}$ Therefore, a comparison of jet spreading with different nozzles should be meaningful only when done at the same Mach number and preferably based on the measurement of the whole flow field. The objective of the present study is to conduct such a comparative evaluation.

In the continuing effort to increase jet mixing and spreading, various other methods, e.g., application of vortex generators in the form of 'tabs', are also under investigation. ${ }^{11-16} \mathrm{~A}$ tab is a small protrusion placed at the jet nozzle exit which produces counter-rotating streamwise vortex pairs. The streamwise vortex pairs, depending on their number, sense and strength, can have a profound impact on the jet spreading. The effect will be summarized in the appropriate context. In the present comparative evaluation, two tab configurations are also included. Both configurations, to be described further shortly, exhibited significant increase in the jet spreading. ${ }^{12,14}$

The bulk of the data, presented in the following, pertain to a fixed subsonic and a fixed supersonic condition. In addition, jet spreading characteristics are evaluated as a function of Mach number for limited cases. In particular, the various stages of screech at the supersonic condition, occurring with varying Mach number, affect the jet spreading significantly. This is explored for the different nozzle geometries. Furthermore, since the use of the tabs is accompanied by a thrust penalty which is of utmost importance in many applications, ${ }^{17,18}$ thrust loss is also measured and analyzed for all the cases.

\section{Experimental Method}

Most of the experiments were carried out in a small jet facility. ${ }^{12}$ Compressed air supplied through one end of a plenum chamber, fitted with flow conditioning units, exited through a $3.81 \mathrm{~cm}$ diameter 'nipple' on the opposite end. The supply air had a total temperature approximately equal to the ambient temperature. The different nozzles could be attached to the nipple. All asym- metric nozzles were machined from solid cylindrical blocks of aluminum by electrical discharge machining (EDM). The interior along the major and the minor axes for each nozzle was contoured according to fourth order polynomial fits. The rest of the interior was faired smoothly. In all cases the flow always converged, and entered and exited the nozzle approximately axially. The equivalent diameter (D) was the same for all the asymmetric nozzles and was $1.47 \mathrm{~cm}$. The circular nozzle was fabricated earlier, and had an exit diameter of $1.27 \mathrm{~cm}$. The general interior shape of each nozzle is shown schematically in Fig. 1(a). All had 'end walls' to facilitate easy installation of the tabs at desired locations.

The asymmetric nozzles included a 3:1 elliptic, a 3:1 rectangular and a generic 6-lobed case. The two tab configurations were: four equally spaced tabs with the circular nozzle and two large tabs spanning the narrow edges of the 3:1 rectangular nozzle. Thus, altogether six nozzle geometries were considered, and these are shown schematically in Fig. 1(b). The tabs used were the 'delta tabs', 12 having triangular shapes with the base on the nozzle wall and the apex leaning downstream at $45^{\circ}$. The

(a)

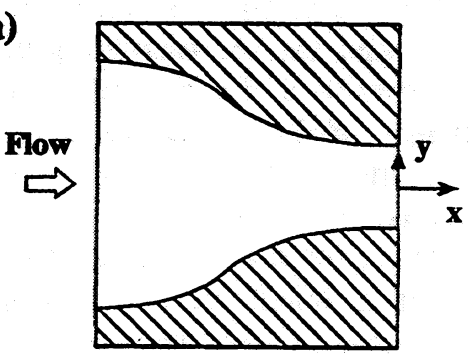

(b)
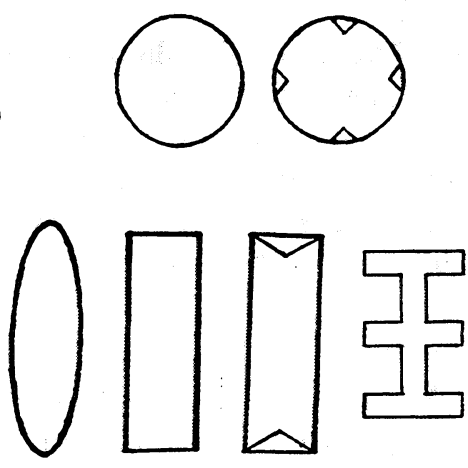

Fig. 1 Schematics of (a) nozzle interior shape and (b) exit geometries of four nozzles with and without tabs. 
tab sizes were such that the area blockage due to each was about $2 \%$ of the nozzle exit area for the circular case (total blockage being about $8 \%$ ), and about $6 \%$ for the rectangular case (total blockage being about $12 \%$ ). These tab configurations were selected after conducting parametric studies designed to yield a large increase in the downstream jet spreading. ${ }^{12,14}$

The mass flux measurements were made at various downstream locations by standard total pressure surveys with a $0.76 \mathrm{~mm}$ (o.d.) Pitot tube. The measurements were conducted for a fixed subsonic $\left(M_{\mathrm{f}}=0.3\right)$ and a fixed supersonic $\left(M_{\mathrm{J}}=1.63\right)$ condition. Since the nozzles were convergent, underexpanded conditions existed in the supersonic regime. The jet Mach number $M_{J}$ in that regime was defined simply as that which would be achieved had the flow expanded fully for a given $\mathrm{P}_{\mathrm{T}} / \mathrm{p}_{\mathrm{A}}$. With the supersonic jets, the measurements were done far enough downstream so that the flow was subsonic everywhere. This was done to avoid measurement errors and probe interference that are typically encountered in supersonic flows.

Throughout the data acquisition, the plenum pressure and ambient pressure were monitored and data normalization was done according to current conditions. Sufficient averaging time was allowed to ensure good data repeatability. The mass flux results were obtained by integration of the Pitot probe survey data over the cross sectional plane. It should be noted that there is subjectivity in the calculation of mass flux. Crow and Champagne $^{19}$ eloquently stated, "The notions of volume flux and entrainment are creatures of theory, in the case of a jet, rather than experiment." This is because the integrand does not fall off to zero in the surrounding potential flow region and, "...the volume flux in the induced potential flow is infinite." In all previous experiments, therefore, various criteria were followed to discriminate the vortical flow from the surrounding potential flow. Crow and Champagne did this for their hot wire data by plotting the integrand, radius times axial velocity, and visually identifying the potential tails of the flux profiles. Note furthermore that both hot wire and Pitot probe measurements involve errors on the outer periphery of the jet due to large turbulence and flow angularity. (With a single hot wire, the measured u-profile in the potential tail involves slowly diminishing but erroneous positive values primarily due to radial entrainment velocity. In data obtained with a Pitot probe, aligned with the jet axis, small sub- ambient values are erroneously read in the tail region, again, due to radial entrainment velocity.)

In view of these difficulties, the criterion followed here simply involved truncating the integration where the measured Mach number dropped below 1\% of the local centerline Mach number. In any case, because of the subjectivity noted in the foregoing, it is felt that specifying a margin of error in the mass flux data is not meaningful. However, with the given truncation criterion, care was taken such that the mass flux data were repeatable within $\pm 4 \%$ for the supersonic conditions, the repeatability being better at upstream locations where the velocities were higher. At the subsonic conditions, because of smaller dynamic pressures, the data scatter was larger and about $\pm 8 \%$. The error in the thrust measurement was estimated to be well within $\pm 1 \%$.

Limited data on the vorticity field for an incompressible case are also presented. The measurement technique will be briefly described with the data. Phase averaged unsteady flowfield data were also obtained for the screeching conditions. The periodic pressure fluctuations were measured as a function of phase over a cross sectional plane of the jet. These measurments were made with a $2.34 \mathrm{~mm}$ diameter pressure transducer (ENDEVCO 8507C-50). The probe measured fluctuating total pressure with sufficient frequency response (manufacturer specification about $200 \mathrm{kHz}$ ). However, the phase-averaged measurements could only be done at locations rather close to the nozzle exit where the core flow was still supersonic. Limited surveys showed that the periodicity in the flow was essentially lost farther downstream. Thus, these measurements involved some probe interference and the results should be considered qualitative. However, the results quite adequately reveal the mode shapes associated with the screech stages. Further details of data acquisition and errors will be given with the results.

\section{Results and discussion}

Figure 2 shows an iso-surface of mean velocity at the subsonic condition $\left(\mathrm{M}_{\mathrm{J}}=0.3\right)$ for the six cases. The amplitude represented by the iso-surface is $30 \%$ of the local maximum velocity; the plots are based on data at five $x$-stations. The two cross-sectional cuts ('surfers') are located at $x / D=2$ and 19. A visual impression of the jet spreading for each case can be obtained from these data. The major axes of the elliptic and the rectangular nozzles were aligned with the z-axis. For the lobed 

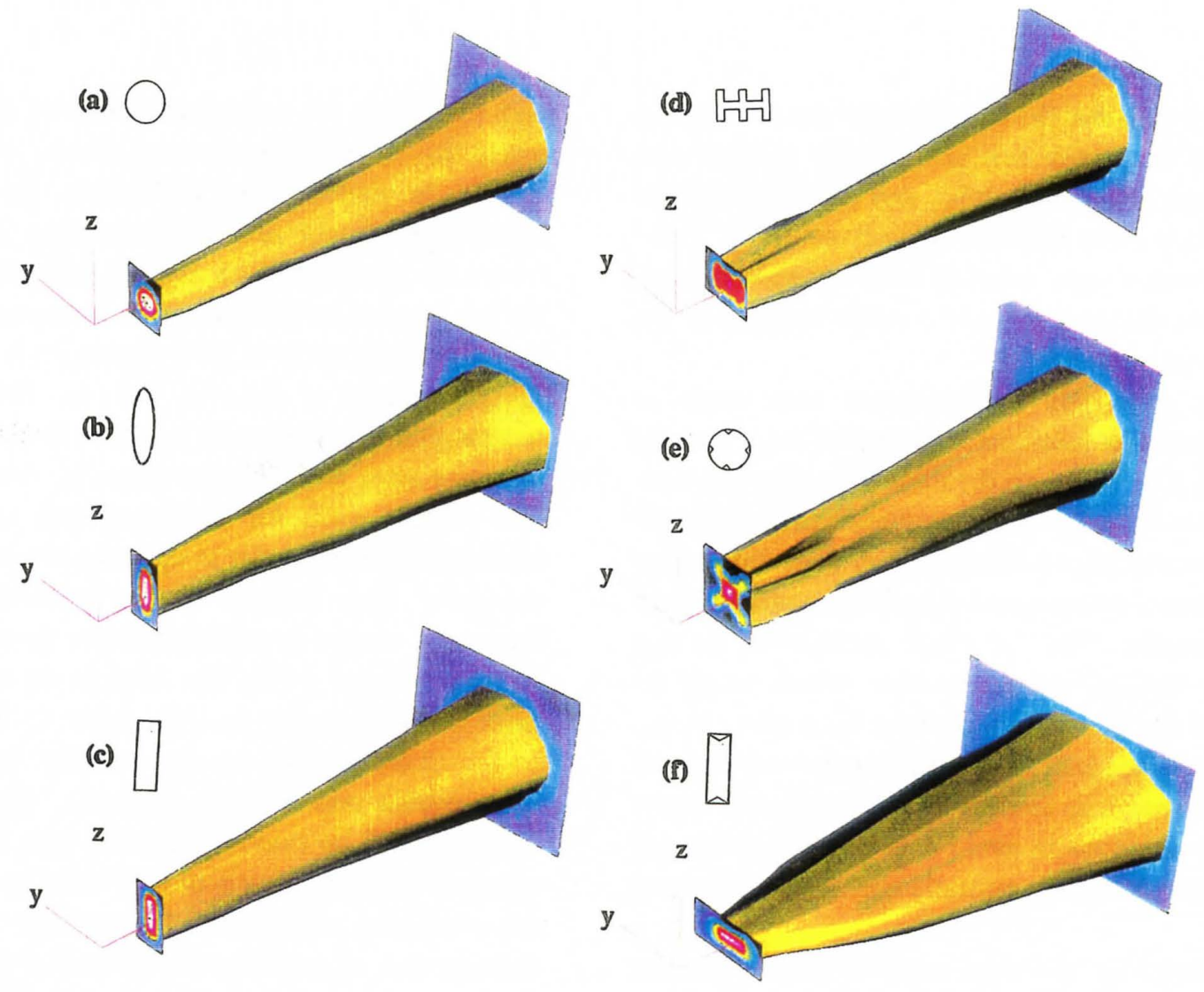

Fig. 2 For $M_{\mathrm{J}}=0.3$, iso-surfaces of mean velocity, $U / U_{\mathrm{MAx}}=0.3$, for the six cases of Fig. 1 (b). Data cover $x / D$ range of 2 to 19.

nozzle, the middle two lobes were aligned with the z-axis. For all cases without the tabs the jet cross section becomes round by the end of the measurement range, and no significant difference in the spreading can be discerned. The two tab cases, in (e) and (f), however, involve noticeable increase in the spreading. Note that for the jet in ( $f$ ), as it can be seen from the 'surfer' on the left, there has already been an 'axis switching' by $x / D=2$. For an asymmetric jet, the axis switching phenomenon is another facet of the spreading characteristics. The dynamics of the large scale vortical structures govern both axis switching and spreading, as to be discussed further in \$3.2.

Corresponding data for the supersonic condition $\left(M_{J}=1.63\right)$ are shown in Fig. 3. In order to save space, the coordinate origin in these data sets have been shown at $x / D=8$. The 'surfer' on the right in all cases is at $x / D=30$, the left 'surfer' locations are indicated in the figure caption. For some cases the measurements were not made at $\mathrm{x} / \mathrm{D}=10$ because the core flow was supersonic. Clearly, the elliptic, the rectangular, and the tab cases result in more spreading. One also finds that unlike at the subsonic condition (Fig. 2), the rectangular and the elliptic jets (Fig. 3b and 3c) have undergone clear axis switching at the supersonic condition. Surprisingly, the lobed nozzle involves minimal spreading. These points are discussed further in the following. Note that the jet spreading in Figs. 3(b), (c) and (f) may appear somewhat exaggerated due to the viewing angle.

The streamwise variation of the maximum velocity for the six subsonic cases of Fig. 2 is shown in Fig. 4. Consistent with the overall jet spreading seen in Fig. 2, one finds the velocity decay to be the fastest for the rectangular jet with two tabs. As expected, the slowest decay occurs for the circular jet without tab. It is noteworthy that the circular jet with four tabs decay faster than any of the three asymmetric cases. Similarly, the variations of the maximum Mach number for the supersonic cases of Fig. 3 are shown in Fig. 5. Again, consistent with jet spreading seen in Fig. 3, the Mach number decay is found to be the slowest for the lobed nozzle and the fastest for the rectangular nozzle with two tabs. 

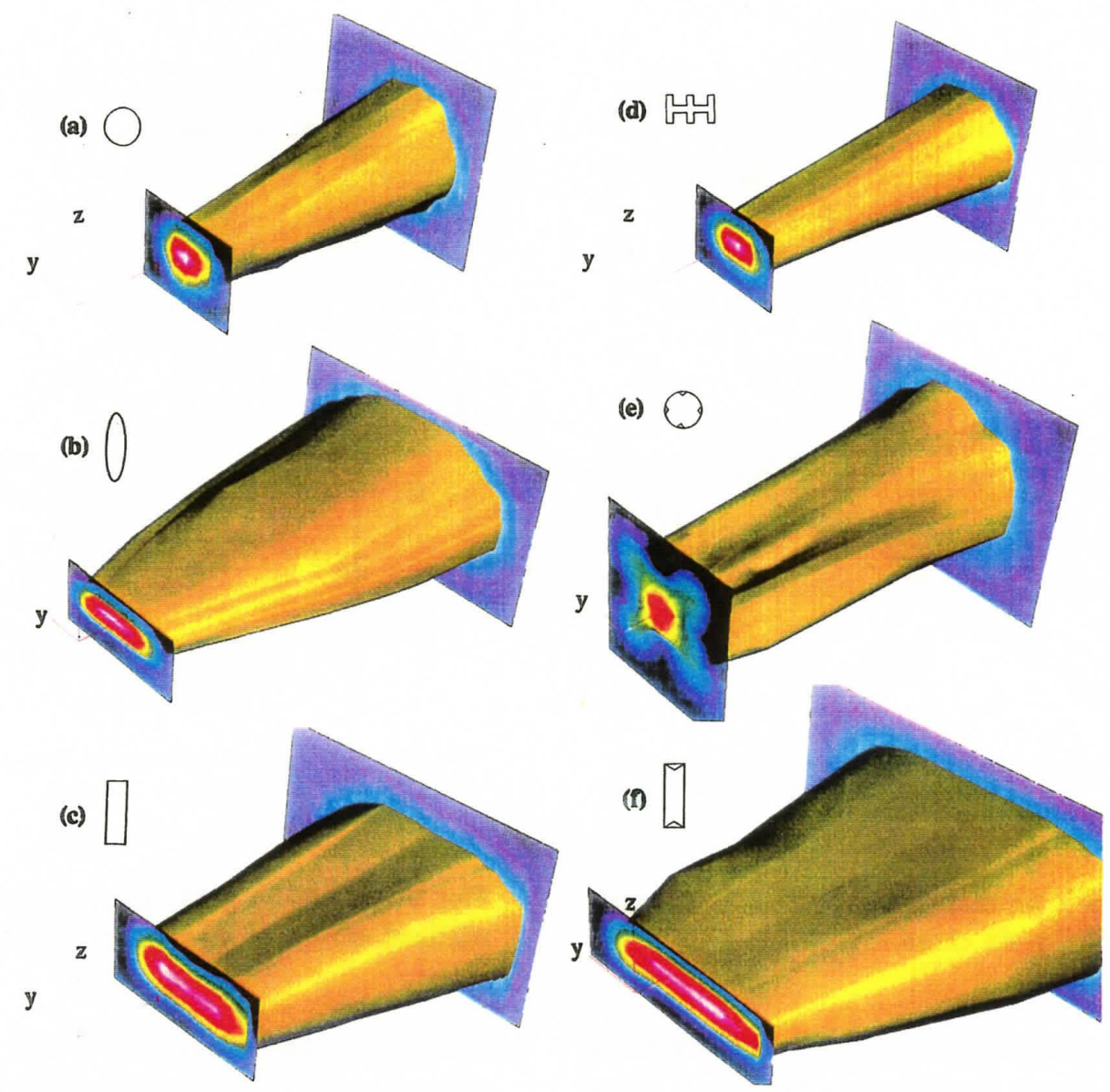

Fig. 3 For $M_{J}=1.63$, iso-surfaces of Mach number, $M / M_{M A x}=0.3$, for the six cases. Data cover $x / D$ range of 14-30 in (a), (c) and (d), and 10-30 in the rest of the cases. Co-ordinate origin is at $x / D=8$.

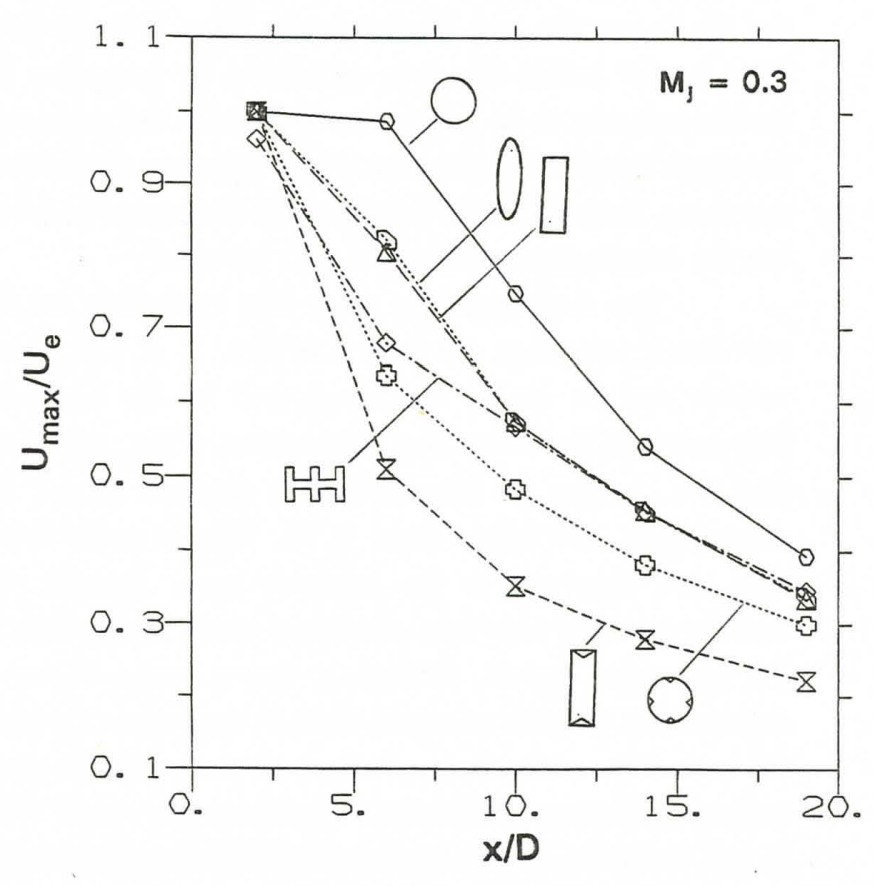

Fig. 4 For $M_{\mathrm{J}}=0.3$, streamwise variations of maximum mean velocity for the six cases.

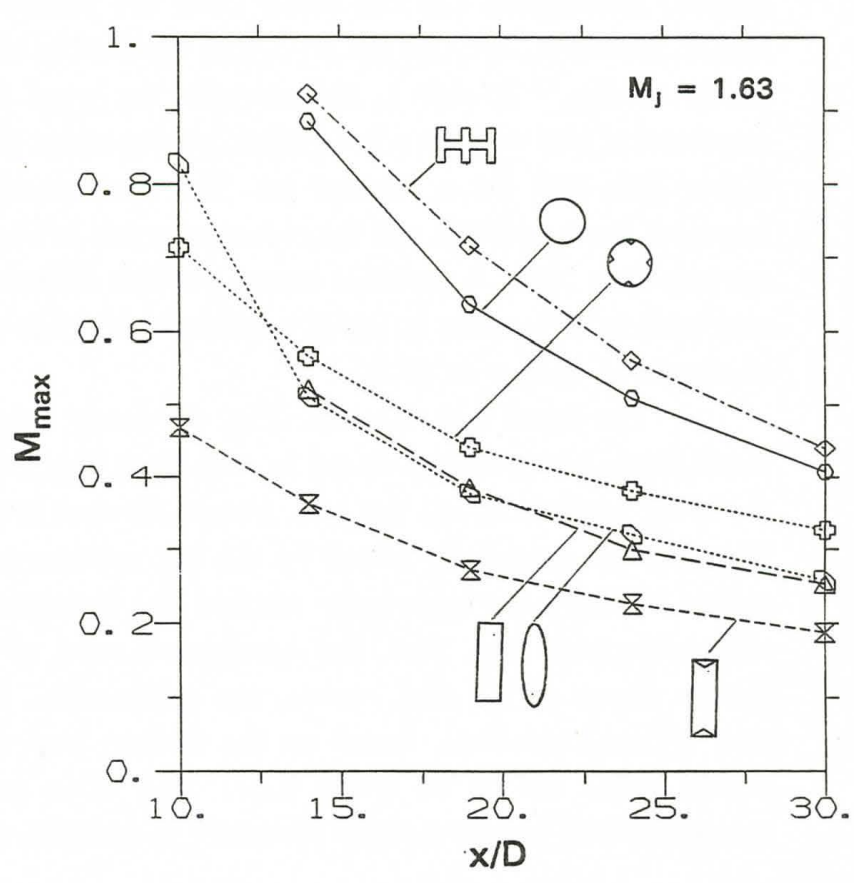

Fig. 5 For $M_{J}=1.63$, streamwise variations of maximum Mach number for the six cases. 


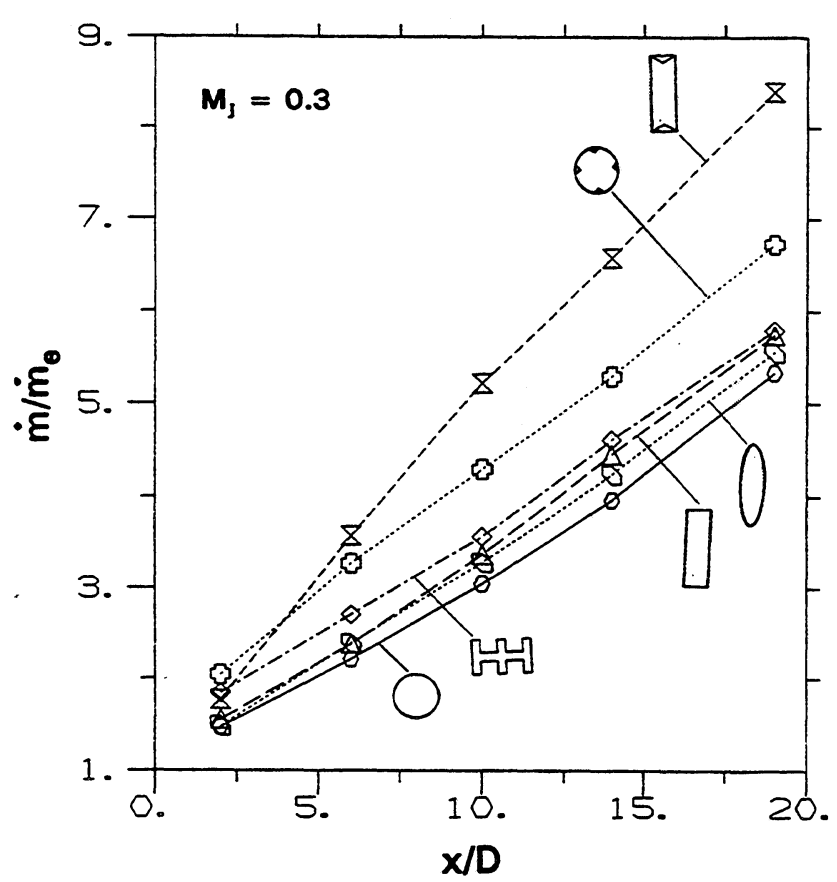

Fig. 6 For $M_{\mathrm{J}}=0.3$, streamwise variations of normalized mass flux for the six cases.

Normalized mass flux variations with streamwise distance for the subsonic cases are shown in Fig. 6. These variations correspond to the Mach number decay shown in Fig. 4, a larger increase in the former is accompanied by a faster decay in the latter. The fluxes for the circular jet are the smallest at all $\mathrm{x}$. Corresponding fluxes for the elliptic, rectangular and the lobed cases are somewhat higher. However, they are not as high as reported in some earlier studies. ${ }^{1,2}$ In ref. 1 , for example, the mass flux measured at $x / D=5$ for a $2: 1$ elliptic jet was about $55 \%$ higher than that for a circular jet. The corresponding increases for the elliptic and the rectangular jets in Fig. 6 are less than 10\%. A possible reason for this difference may trace to differences in initial conditions, and this will be discussed further in \$3.3.

The cases with the tabs (Fig. 6) clearly exhibit much larger jet spreading. It can be seen from Fig. 2 that the cross sections of all the jets, except the one in (f), have become essentially round by the last measurement station. These have apparently reached the asymptotic, self-similar state and, thus, the entrainment rates, given by the slopes of the flux curves, are comparable. The rate, $\left(\left(D / \dot{\mathrm{m}}_{\mathrm{F}}\right) \cdot(\partial \dot{\mathrm{m}} / \partial \mathrm{x})\right)$, based on the farthest two data points, turns out to be about 0.27 . This is in good agreement with previous round jet data. ${ }^{19}$ In contrast, the

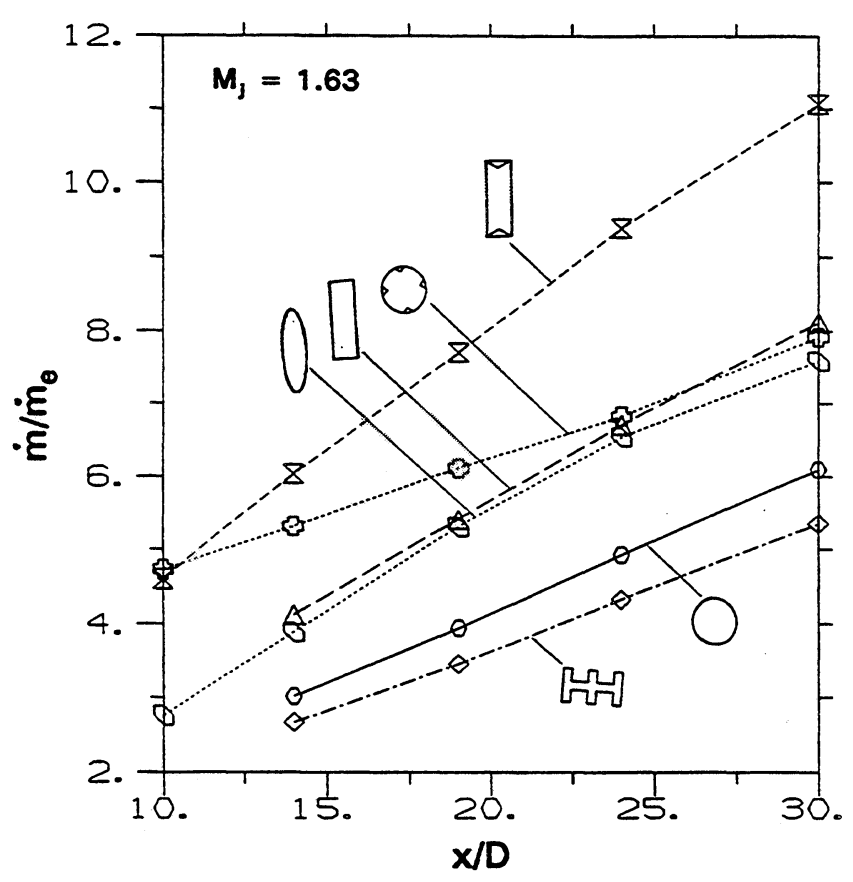

Fig. 7 For $M_{\mathbf{J}}=1.63$, streamwise variations of normalized mass flux for the six cases.

rectangular jet with tabs in (f) is still undergoing entrainment at a higher rate yielding the higher fluxes. One might expect that eventually this jet would also attain a round cross section. When that happens farther downstream, the entrainment should reach the same asymptotic rate as exhibited by the others. (In obtaining the data for the tab cases, the area blockage was taken into account. That is, the initial flux was calculated by assuming the nozzle exit area to be less by the amount of the geometric blockage imparted by the tabs. Note that even if this were not done, the normalized fluxes would be somewhat lower but the trends discussed would still be valid).

The corresponding mass flux variations for the supersonic condition are shown in Fig. 7. A few observations can be made, and these will be the basis for discussion in the following subsections. First, one finds that the circular jet has attained a constant slope, the entrainment rate being about 0.18 . This is much lower than that observed in the subsonic case. Second, the circular jet with four tabs exhibits a much higher flux in the upstream region, indicating that a more vigorous entrainment process occurred farther upstream. Towards the end of the measurement range, however, the jet cross section in this case has become approximately round (Fig. 3) and the slope of the flux curve has become comparable 
to that of the corresponding no tab case. Third, as with the subsonic case the rectangular jet with two tabs is found to involve the most vigorous entrainment within the measurement range. Fourth, the flux values for the lobed nozzle are found to be even lower than those for the circular nozzle! Finally, one finds that for both the elliptic and the rectangular jets the fluxes are significantly higher than the circular jet. This is in contrast to the subsonic case where the corresponding difference was smaller. These points are further discussed next.

\subsection{Asymptotic entrainment rate for initially compressible} jets:

In regards to the lower entrainment rate for the circular jet at the supersonic condition, the following can be said based on similarity reasonings. Ideally, all jets under consideration, with any nozzle geometry, subsonic or supersonic, should eventually become round and selfsimilar with incompressible flow. When that happens, a local length scale $\left(D_{H}\right)$ should vary linearly as $x$ and a local velocity scale $\left(U_{c}\right)$ inversely as $x .^{20}$ It follows that the streamwise mass flux $\dot{m}$ should equal to $p_{A} C_{1} \cdot U_{C} \cdot D_{H}{ }^{2}$, which should vary linearly as $x$; here $\rho_{A}$ is the ambient fluid density and $C_{1}$ a constant. For the asymptotic region, the functional relationships for $D_{H}$ and $U_{C}$ can be written as: $D_{H} / D=C_{2} \cdot(x / D)$, and $U_{C} / U_{E}=C_{3} /(x / D)$; where $C_{2}$ and $C_{3}$ are constants. It follows from these expressions that the entrainment rate, $\partial\left(\dot{\mathbf{m}} / \dot{\mathrm{m}}_{\mathrm{F}}\right) / \partial(\mathrm{x} / \mathrm{D})$, equals a constant, $C^{\prime}=C_{1} \cdot C_{2}^{2} \cdot C_{3} \cdot(4 / \pi)$. Experimental data for incompressible jets yield a value of this constant in the approximate range of $0.26-0.3 .{ }^{19,21-23}$

For the asymptotic region of initially compressible jets, the functional relationships for $D_{H} / D$ and $U_{C} / U_{E}$ should still hold. It then follows, $\partial\left(\dot{m} / \dot{\mathrm{m}}_{\mathrm{E}}\right) / \partial(\mathrm{x} / \mathrm{D})=$ $\left(\rho_{A} / \rho_{E}\right) C_{1} \cdot C_{2}^{2} \cdot C_{3} \cdot(4 / \pi)$. The constant on the right hand side here differs from that for the incompressible case by a factor of $\left(P_{\mathrm{A}} / \rho_{E}\right)$, which for $M_{J}=1.63$ turns out to be about 0.65 . This qualitatively agrees with the lower slope of 0.18 at the supersonic condition compared to the value 0.27 observed for the subsonic condition. Here, it is pertinent to mention the work of Ref. 24. The functional dependence of $U_{C} / U_{E}$ on $x / D$ was investigated based on data from earlier experiments. Correlation of the data exhibited a mild dependence of $U_{C} / U_{E}$ also on $\left(\rho_{A} / \rho_{E}\right)$. Such a dependence would yield a different expression for the entrainment rate. However, the data examined in Ref. 24 covered the near flow fields. It appears that the far asymptotic regions of especially high Mach number jets were not covered in the investigation.

The validity of the expression for the asymptotic entrainment rate for initially compressible jets was further examined with the axisymmetric nozzle. Normalized mass flux curves as in Fig. 7 were obtained for different Mach numbers $\left(M_{\mathrm{J}}\right)$. These data are presented in Fig. 8. The slopes calculated from the farthest two data points in each curve, and the resultant constants in the expression for $\partial\left(\dot{m} / \dot{m}_{\mathrm{f}}\right) / \partial(\mathrm{x} / \mathrm{D})$, are listed in Table 1 . It is clear that the value of the constant, after the 'compressiblity effect' is taken into account, as shown in the third column, is within reasonable scatter. Thus, the expression provided in the previous paragraph for the asymptotic entrainment rate appears to be valid. Note that the rate for $M_{J}=1.38$ case shows the largest deviation. As to be discussed in \$3.3, the jet at $M_{\mathbf{J}}=1.38$ involves screech with a pronounced flapping motion. The jet cross section, even on the time-averaged field, is somewhat noncircular which persists within the measurement range. Thus, as with the asymmetric cases (Fig. 7), the jet in this case may not have reached the asymptotic state and the higher entrainment rate is likely to be real.

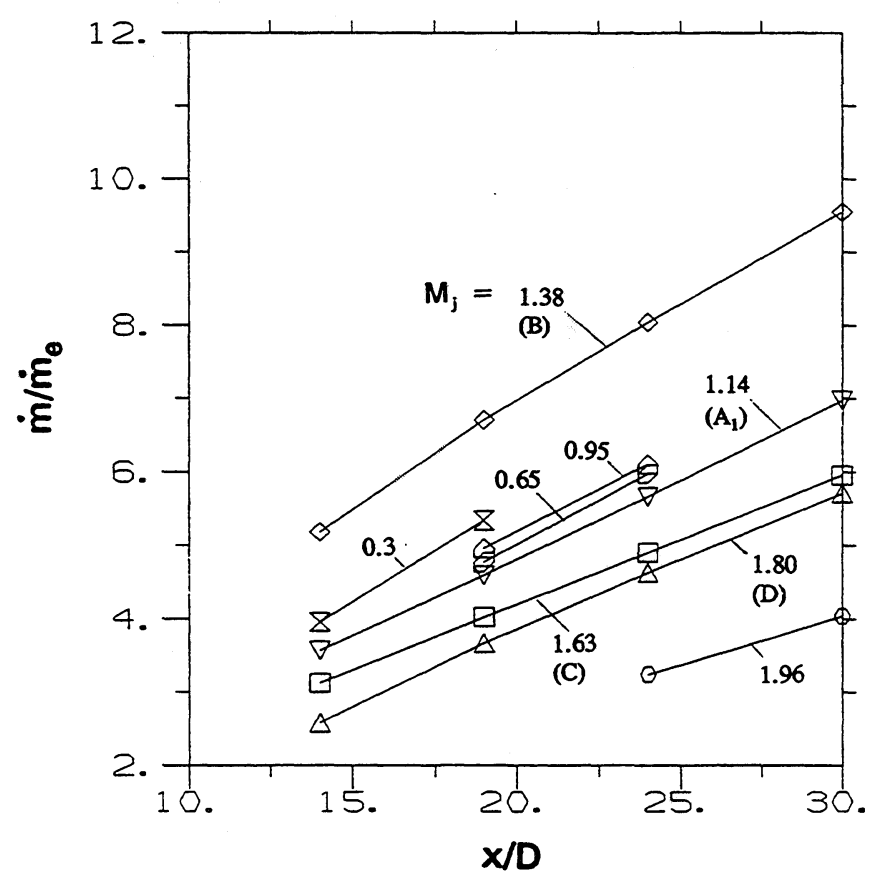

Fig. 8 For the circular nozzle, streamwise variations of normalized mass flux at different $M_{\mathbf{J}}$ (letters in parentheses indicate screech stages). 
Table 1 Entrainment rates for the various cases of Fig. 8

$\begin{array}{lll}\mathrm{M}_{\mathrm{J}} & \left(p_{\mathrm{A}} / \rho_{\mathrm{E}}\right)^{*} \mathrm{C}^{\prime} & \mathrm{C}^{\prime} \\ 0.30 & 0.274 & 0.279 \\ 0.65 & 0.241 & 0.261 \\ 0.95 & 0.229 & 0.271 \\ 1.14 & 0.220 & 0.277 \\ 1.38 & 0.251 & 0.347 \\ 1.63 & 0.176 & 0.270 \\ 1.80 & 0.180 & 0.296 \\ 1.96 & 0.134 & 0.238\end{array}$

Note, again, that the inferences made in the foregoing pertain to the asymptotic regions of the jets. Some of the asymmetric jets (Figs. 6 and 7) obviously have not reached that state within the measurement range. It should also be noted that the effect considered here is not the same as the well known compressibility effect causing a reduction in mixing layer spreading. ${ }^{10}$ The latter effect would prolong the length of the developing region of the jet. Here, the lower slope measured in the asymptotic region occurs simply due to the fact that the normalizing parameter, $\dot{\mathbf{m}}_{\mathrm{r}}$, is ralatively large for the initially compressible case because of higher initial densities. Furthermore, it is worth noting in passing that the effort to increase jet spreading using various techniques, such as tabs, asymmetric nozzle shaping, screech, multiple jet resonance, etc., pertain only to the developing regions. Far enough downstream, the same asymptotic behavior may be expected in all cases. However, it is clear that the developing region can be very significantly affected by these different techniques which is of great importance in practical applications.

\subsection{Effect of tabs and streamwise vorticity on spreading:}

The second and third points made with Fig. 7 concern the increased entrainment caused by the tabs. The observed increase is due to the action of streamwise vortex pairs produced by the tabs. This becomes clear from the results presented in Refs. 12 and 14, which are briefly summarized here.

A tab produces a pair of counter-rotating streamwise vortices with a sense of rotation such that, between the two vortices, ambient fluid is ingested into the core of the jet. This is referred to as an 'in-flow' vortex pair. Four equally spaced tabs on the circular nozzle, therefore, produce four such in-flow pairs. Vortex induced velocities at first make all four pairs move towards the jet axis. However, this motion is restricted and, shortly downstream, vortices from adjacent pairs form four 'out-flow' pairs. Each of these pairs causes an ejection of jet core fluid into the ambient. The induced motion also propel the vortex pairs themselves away from the jet axis. The vigor of the dynamics depends on the strength of the vortices which in turn depends on the tab geometry. For the present tab configuration, the dynamics are indeed quite vigorous which cause the observed increase in jet spreading. In Ref. 12, it was also shown that for the given size and geometry, four tabs increased the spreading optimally. When six tabs were used, for example, amalgamation among the vortices occurred, dropping the number of outflow pairs and reducing the resultant jet spreading.

With the asymmetric jets the tab effect is more complex. This is because these jets typically already contain streamwise vortices due to secondary flow within the nozzle. Depending on the sense and strength of these, and the vortices produced by the tabs, the interaction can be different. Let us examine the streamwise vorticity distribution for the present tab case with the rectangular nozzle. Hot wire measurements were conducted with a nozzle which was geometrically similar to the present rectangular one but about five times larger in $D$. The larger size was necessary from probe resolution considerations. Two $\mathrm{X}$-wire probes were used to obtain the distributions of the lateral velocities ( $v$ and $w$ ) on a cross sectional plane, the gradients of which provided streamwise vorticity $\left(\omega_{x}\right)$. Further details of the measurements can be found in Ref. 14 and those are not repeated here for brevity.

The vorticity data for the tab case are compared with the no tab case in Fig. 9. Two iso-surfaces $\left(\omega_{x} D / U_{E}\right.$ $= \pm 0.08)$ are shown. Looking from downstream, darker and lighter surfaces represent clockwise and anti-clockwise sense of rotation, respectively. The flowfield without tabs can be seen to contain two out-flow vortex pairs occurring on the narrow edges. The two pairs would move away from the jet axis, and thus tend to keep the jet cross section elongated in the major axis plane. By $x / D=$ 8, the range covered in Fig. 9, these vortices diminish in strength and the jet cross section becomes almost round (Fig. 2c). When the tabs are applied, two strong in-flow pairs are produced on the narrow edges. These overwhelm the naturally occurring vortices and completely dominate 


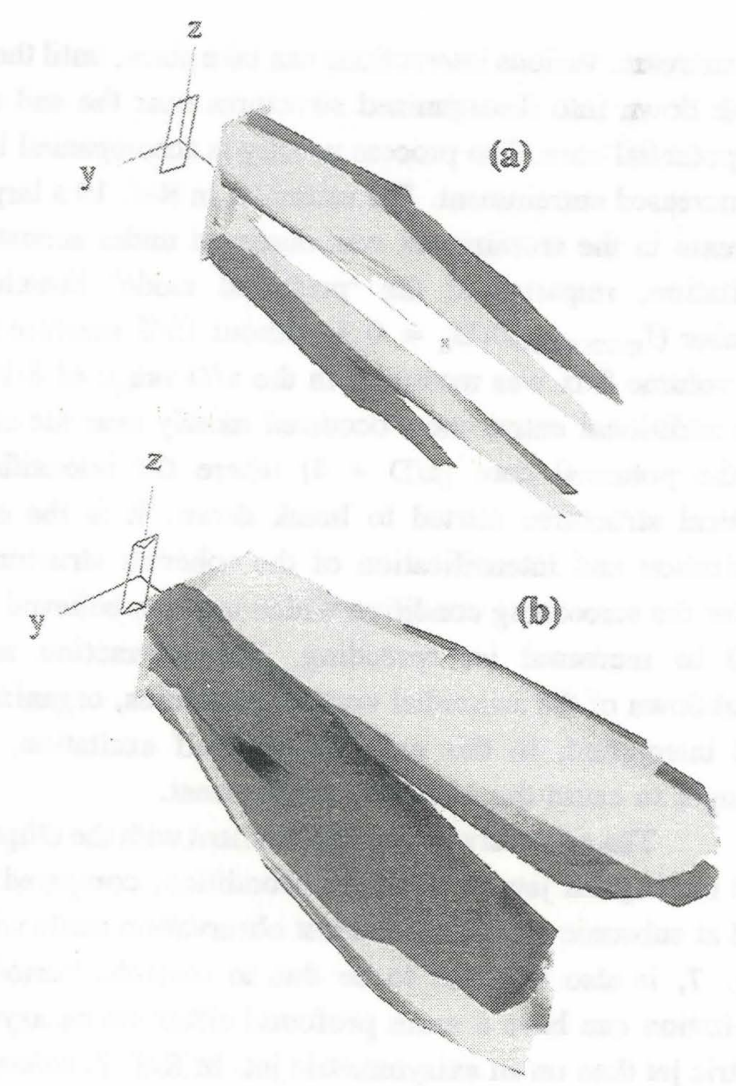

Fig. 9 Time-averaged streamwise vorticity, $\omega_{\mathrm{X}} \mathrm{D} / \mathrm{U}_{\mathrm{E}}=$ \pm 0.08 iso-surfaces, for a 3:1 rectangular nozzle: (a) no tab, (b) two tabs.

the flow. The in-flow pairs first move towards the jet axis, and thus, cause the rapid axis switching. Thereafter, the vortices rearrange to form two out-flow pairs which continue to cause the lateral spreading in the direction of the minor axis. It should be mentioned here that the axis switching could be achieved with much smaller size tabs, (or prevented by putting the tabs on the long edges; see Ref. 14). However, the enormous lateral spreading, as observed in Figs. 2, 3, occurred only when the tab base spanned the entire narrow edge of the nozzle.

Thus, it is amply clear that the effect of the tabs occurs primarily due to the dynamics of streamwise vortex pairs. Even though the tabs produce in-flow pairs, the vortices rearrange to form out-flow pairs which cause the spreading. For the asymmetric jets the process also readily affects the axis switching phenomenon. It appears that generating an optimum number of out-flow vortex pairs with appropriate strength may hold a key to achieving even larger jet spreading.
Note that the dynamics discussed in the foregoing pertain to the time-averaged vorticity field. Instantaneously the vortices can be much stronger and the dynamics more vigorous. ${ }^{14}$ The reorientation of azimuthal vorticity gives rise to streamwise vortex pairs in the unsteady field which also contribute to jet spreading. Liepmann and Gharib $^{25}$ convincingly demonstrated the important role played by instantaneous streamwise vortices in the entrainment process. The role of azimuthal vorticity, on the other hand, especially in the near field of an asymmetric jet, can also be quite significant for jet spreading as well as axis switching, and this is addressed in the next section.

\subsection{Effect of screech on jet spreading:}

The fourth point made with Fig. 7 was that the least amount of spreading was exhibited by the lobed nozzle. An underlying concept for a lobed nozzle is to stretch the mixing layer region exposed to the ambient so that there is more mixing and entrainment. Thus, the result that the lobed nozzle produced less jet spreading compared to even the circular nozzle came as a total surprise. Soon the reason became apparent. At $M_{\mathbf{J}}=$ 1.63 , all the jets (without tabs) involved screech, whereas there was no screech with the lobed nozzle. It may be reasoned that the presence of screech with the other nozzles and the absence thereof with the lobed nozzle caused the difference.

First, the far field noise spectra for the six cases under consideration are shown in Fig. 10. These data were obtained with a microphone located approximately 70D away from the jet axis and about $110^{\circ}$ from the downstream axis. For ease of comparison the data are shown in three pairs. The fundamental screech component at about $9 \mathrm{kHz}$ can be clearly seen for the circular jet. This is eliminated when the tabs are applied. Both the rectangular and the elliptic nozzles are also characterized by screech noise. Use of the tabs does not completêly eliminate screech from the rectangular nozzle but the amplitudes are reduced drastically together with a shift in the frequencies. It is also clear that the lobed nozzle does not involve any screech noise. This is true throughout the Mach number range covered in the study.

Screech is a phenomenon that occurs in imperfectly expanded jets due to the interaction of coherent azimuthal vortical structures with the standing shockexpansion cells. ${ }^{26-30}$ The vortical structures are organized 


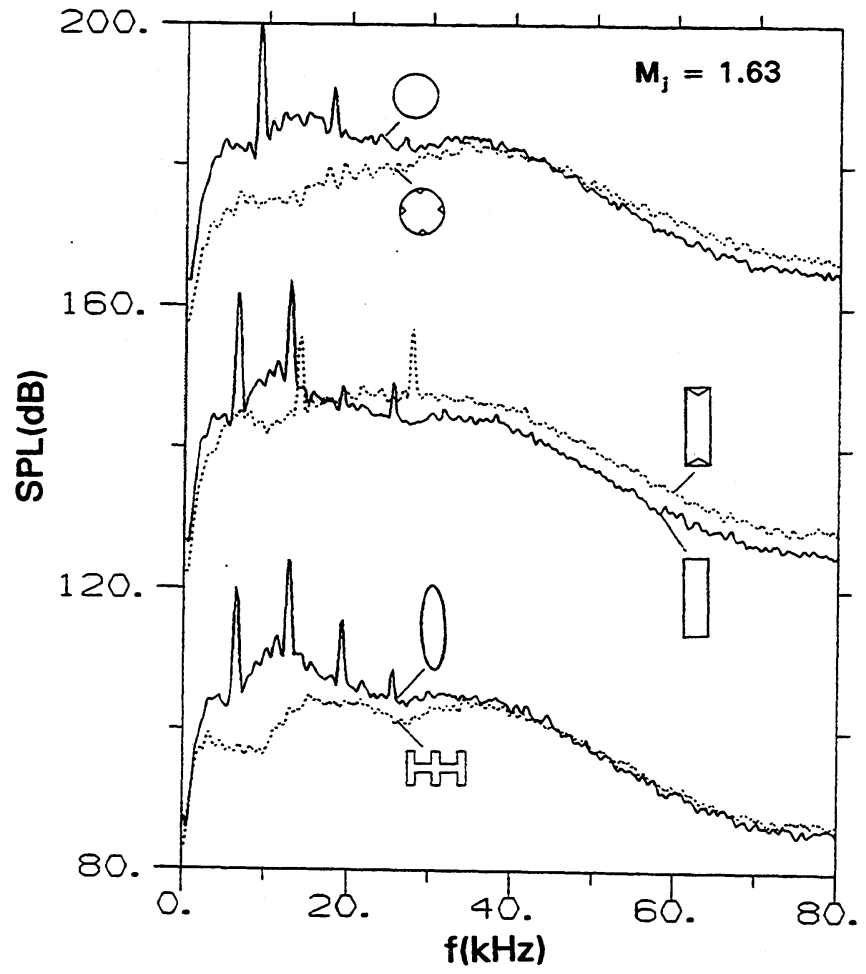

Fig. 10 Far-field noise spectra for the six cases; the three pairs of traces are staggerred successively by one major ordinate division.

and rendered periodic through a feedback loop. The complete mechanism remains unclear, but the interaction also results in the emission of a tone "...of definite frequency and high intensity".27

Screech is also accompanied by an increased jet spreading. The first known investigation addressing this effect for a circular jet is that of Glass. ${ }^{31}$ The generation of screech in Glass's study caused a 'precipitous drop' in the 'impact pressure' measured on the jet axis, clearly indicating a significant increase in the spreading of the jet. Later on, the effect was further investigated by Sherman et al. ${ }^{32}$, and, for a rectangular jet, by Krothapalli et al. $^{33}$

Based on past studies on the effect of artificial excitation on incompressible jets, the following comments can be made on the likely mechanism for the effect of screech on jet spreading. A primary effect of artificial excitation, when imparted at an appropriate frequency, is to organize and intensify the coherent vortical structures. For the case of a circular jet, the excitation causes the azimuthal vorticity to periodically roll up into toroidal structures. These structures enlarge as they convect downstream, various interactions can take place, until they break down into disorganized structures near the end of the potential core. The process usually is accompanied by an increased entrainment. For example, in Ref. 19 a large increase in the entrainment was observed under acoustic excitation, imparted at the 'preferred mode' Strouhal number $\left(f_{E X c t a t i o N} D / U_{E}=0.3\right)$. About $15 \%$ increase in the volume flux was measured in the $x / D$ range of 8-10. The additional entrainment occurred mostly near the end of the potential core $(x / D-4)$ where the intensified vortical structures started to break down. It is the organization and intensification of the coherent structures under the screeching condition which are also believed to lead to increased jet spreading. The interaction and breakdown of the azimuthal vortical structures, organized and intensified, in this case, by the self excitation, is thought to cause the increased entrainment.

The relatively higher entrainment with the elliptic and rectangular jets at supersonic condition, compared to that at subsonic condition, the last observation made with Fig. 7, is also believed to be due to screech. Periodic excitation can have a more profound effect on an asymmetric jet than on an axisymmetric jet. In Ref. 2, acoustic excitation of subsonic elliptic jets has been shown to cause a dramatic increase in the jet spreading. Under the excitation there is an organization and intensification of the rolled up asymmetric vortical structures. The self induction and the resultant sequence of contortion of these structures not only cause axis switching, ${ }^{2,14}$ but also result in an increased engulfment of ambient fluid. Again, it is thought that a similar mechanism is in play with the asymmetric jets when screech occurs. This causes the larger increase in the fluxes at the supersonic condition (Fig. 7) compared to the increase at the subsonic condition (Fig. 6). This also explains the axis switching seen in the supersonic cases (Fig. 3) which did not occur in the subsonic cases (Fig. 2).

Recall the difference in the mass flux results between the present asymmetric jets (Fig. 2) and that of Ref. 1, as discussed earlier. It should be recognized that the dynamics of the asymmetric vortex structures described in the previous paragraph is also active in a natural jet (without excitation) because there is natural roll up of the structures. Excitation accentuates the process and thereby increases the entrainment as well as causes the earlier axis switching. The process in the natural case, however, can be vary from jet to jet because there can be 
varying amounts of self excitation depending on the initial and background conditions. At the lower Mach number and Reynolds number, the initial boundary layer was laminar and the core turbulence was lower in the jets of Ref. 1. At $M_{\mathbf{J}}=0.3$ with the present nozzles, the initial boundary layers were nominally turbulent. ${ }^{14}$ Thus, a more organized initial roll up of the azimuthal vorticity in the cited work was likely. This is thought to have caused the larger entrainment, in a similar manner as it occurs under artificial excitation.

The discussion so far has been based on previous studies involving 'plane wave' acoustic excitation. There have been other studies involving excitation with more complex waveforms (subharmonic, helical, combination of modes, etc.). In most cases, artificial excitation at the various modes also cause an increase in the overall jet spreading. ${ }^{34}$ Screech involves self excitation in a variety of modes. The characteristics of these modes and their impact on jet spreading are further explored in the following.

\subsubsection{Unsteady flow characteristics in various stages of} screech: First, let us examine the screech frequency chracteristics. Variations of screech frequency, expressed as Strouhal number, for the circular, elliptic and rectangular jets are shown in Fig. 11. The triangular and

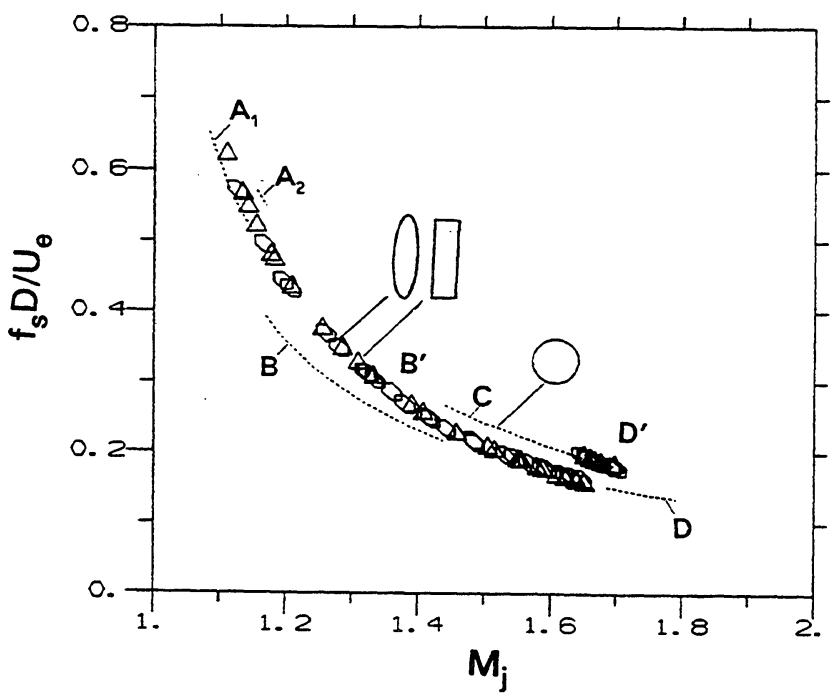

Fig. 11 Nondimensional screech frequency $\left(f_{s}\right)$ versus $M_{\mathrm{J}}$ for the indicated nozzles; letters indicate screech stages. elliptical symbols represent the rectangular and elliptic jets, respectively, while the dotted line represent data for the circular jet. As observed by many previous researchers, ${ }^{27-20,35,36}$ the screech for the circular nozzle is seen to go through different stages as the nozzle pressure ratio (or $\mathbf{M}_{\mathrm{y}}$ ) is varied. These stages involve, as noted in the cited studies and as to be further discussed in the following, flow field oscillations in the axisymmetric (stages $A_{1}$ and $A_{2}$ ), flapping (B and D) and helical (C) modes. Corresponding frequency characteristics for the rectangular and the elliptic jets also show a staging behavior involving one frequency jump within the measurement range. A jump occurs from B' mode to $D$ ' mode around $M_{J}=1.65$ (52 psig) for both the nozzles, with a certain amount of overlap between modes. The nature of the unsteady flow fields in the different screech stages for the different nozzles is further explored by the phase averaged total pressure measurements.

As described in $\$ 2$, the phase averaged data were obtained at a given downstream location over the jet cross sectional plane. The location of measurement was determined for each $\mathbf{M}_{\mathrm{J}}$ for a given nozzle by initial surveys. The choice was dictated by the requirements that the location was far enough downstream in order to avoid probe interference as much as possible, yet sufficiently upstream to ensure coherence in the flow fluctuations. These criteria yielded measurement locations that were generally farther downstream with increasing $\mathbf{M}_{\mathbf{J}}$. Typically, the data were acquired for $13 \times 13$ grid points over the cross sectional plane. The signal from a microphone located near the nozzle exit was used as reference. The phase averaged total pressure, relative to the time averaged total pressure, was measured at each grid point. The data were obtained for nineteen phase points over a cycle and involved averaging over approximately 300 sample functions.

Data for stages $A_{1}, A_{2}, B, C$ and $D$ of the circular nozzle are shown in Fig. 12. The Mach numbers and measurement locations are indicated in the figure caption. For each case, two equal and opposite (positive and negative) iso-surfaces are shown. The viewing angle (same for all the cases) and the iso-surface levels were chosen to demonstrate the nature of the fluctuating field as clearly as possible. The data vividly demonstrate that the mode shapes in the two A stages are axisymmetric. Those in stages $\mathbf{C}$ and D are clearly helical and flapping, respectively. The flow pattern in stage B is also primarily 


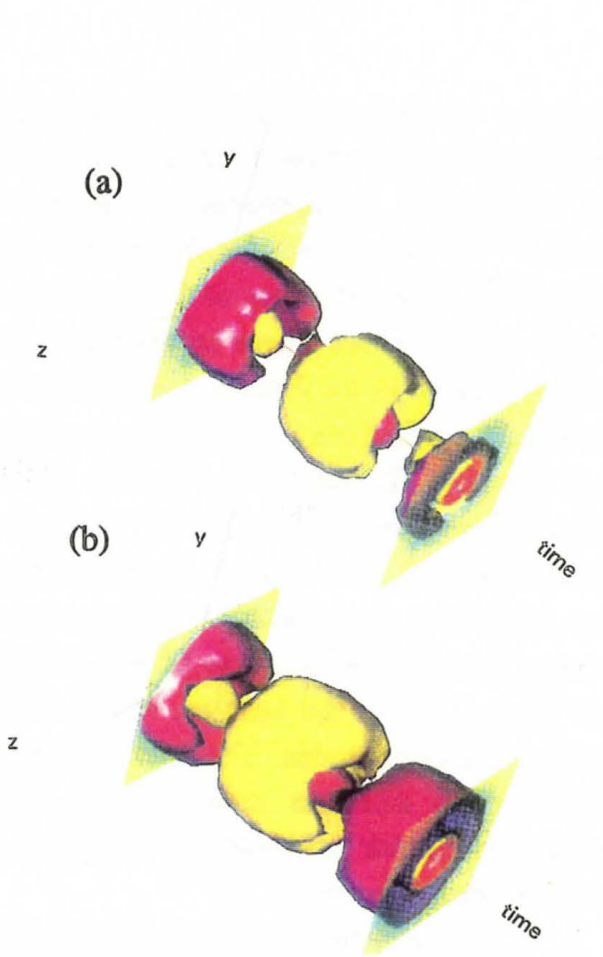

Fig. 12 Phase-averaged unsteady total pressure distributions for the circular jet. Screech stage, $\mathbf{M}_{\mathbf{J}}$ and measurement location ( $x / D$ ) are: (a) $A_{1}, 1.11,4.5$, (b) $\mathrm{A}_{2}, 1.16,4.5$, (c) $\mathrm{B}, 1.37,6$, (d) $\mathrm{C}, 1.63,9$, (e) $\mathrm{D}, 1.81,12$.

flapping but is apparently mixed with a helical pattern. An unsteadiness and switching from helical to flapping oscillation was also inferred for the B mode in the studies of Refs. 35 and 37. As stated before the mode shapes associated with the circular jet screech stages had been inferred earlier in several studies. These were done through correlation measurements in the near acoustic field as well as by schlieren visualization of the flowfield. However, ambiguities can arise in interpreting the results of such measurements. For example, it can be difficult to differentiate the helical mode from the flapping mode. The present results unambiguously confirm the mode shapes and provide a visual impression of the fluctuating flowfield.

The corresponding data for the stages denoted B' and D' (Fig. 11), for the 3:1 rectangular nozzle, are shown in Fig. 13. It is clear that both the stages involve flapping motion about the $\mathrm{x}, \mathrm{y}$ plane. Similar data for the elliptic nozzle (not shown) also yielded essentially the same modal structures.
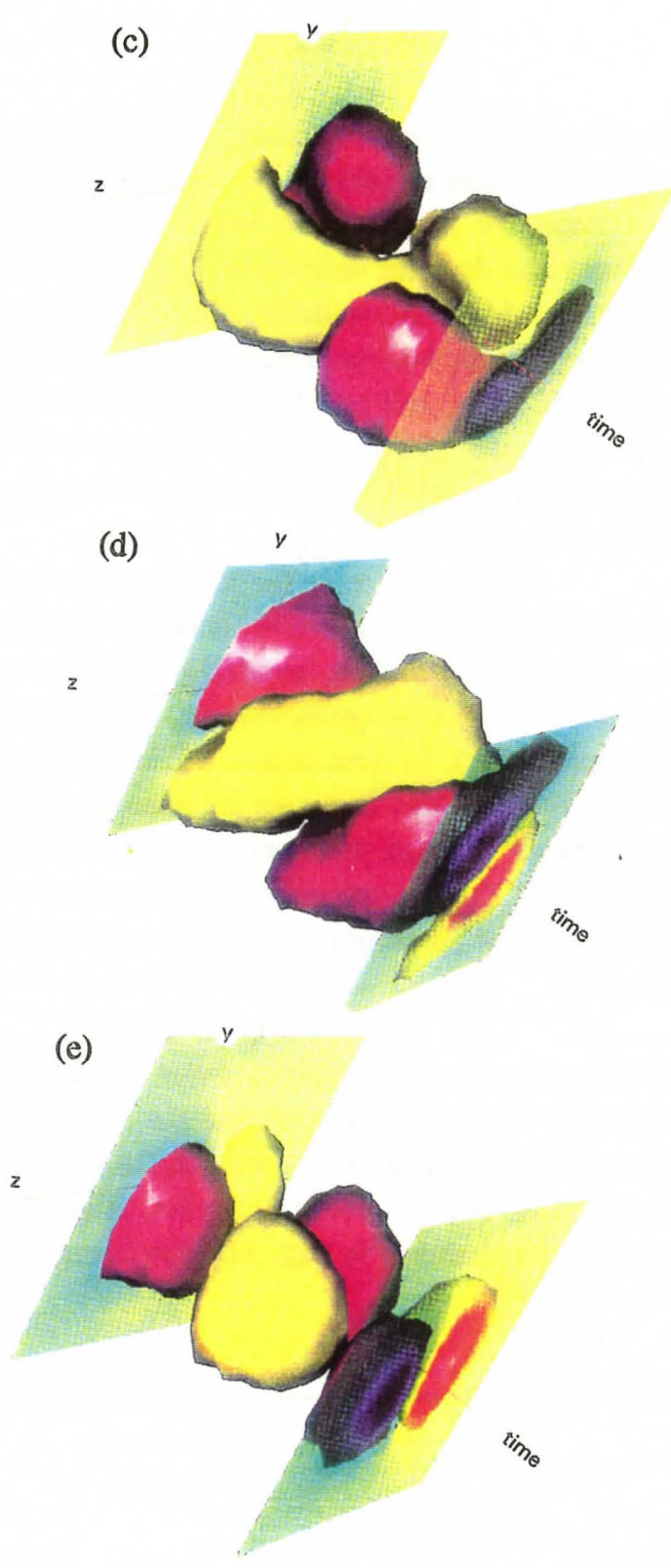

Why does the jet go through a frequency jump from stages B' to D' with seemingly similar unsteady motion? Why does the circular jet go through a jump from $A_{1}$ to $A_{2}$ mode both having axisymmetric unsteady motion? What dictates the other modal structures with the circular nozzle? What chooses the direction of the helical motion as well as the plane about which the flapping motion occurs? These issues, as well as the complete mechanism for the screech noise generation, remain far from clear. These are considered beyond the scope of the present work. (The reader is referred to Ref. 36 for an insight into the unsteady shock motion associated with 


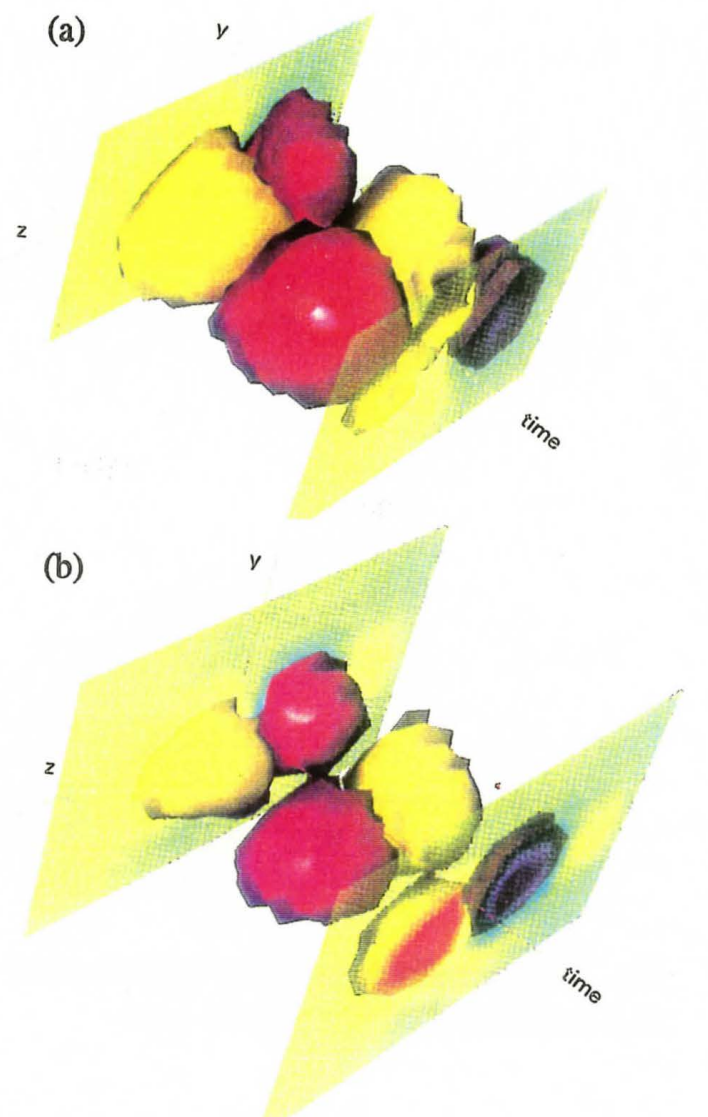

Fig. 13 Phase-averaged unsteady total pressure distributions for the rectangular jet. Screech stage, $M_{J}$ and $x / D$ are: (a) B', 1.63, 6, (b) D', 1.75, 8 .

screech, and to Ref. 38 for the effect of induced screech on the jet via placement of obstacles in the flow. These are parallel studies being conducted in the same laboratory). Here, let us make some further comments on the data of Figs. 12 and 13 before turning attention back to the spreading of the various jets.

The unsteady amplitudes shown in Figs. 12 and 13 are values over the local time averaged values. The maximum peak-to-peak amplitudes are typically about $0.2 \%$ of the local total pressure. These are small compared to common experience with excitation studies of subsonic jets. The red (darker) iso-surface represents regions of positive (higher) total pressure or streamwise velocity. The yellow (lighter) iso-surface represents regions of negative (lower) streamwise velocity. For the axisymmetric modes $\left(A_{1}, A_{2}\right)$ with the circular jet, one would expect the toroidal vortical structure to be located near the middle of the field, where the velocities are higher in the core but lower outside. (Note that an inner red iso-surface in this region is surrounded by a outer yellow iso-surface). Mode C (Fig. 12d) is clearly helical. The location of the corresponding helical vortex tube is not completely clear, but is probably between the red and yellow regions. Mode D can be viewed as due to the superposition of two opposite sense helices causing the flapping motion. A certain plane of flapping is chosen by the jet, presumably, either due to irregularities in the geometry of the upstream hardware or in the recirculating flow in the laboratory. For the rectangular nozzle, the flapping plane is well defined and coincident with the major axis plane of the nozzle. This is clearly seen in both sets of data of Fig. 13.

\subsubsection{Effect of various screech stages on jet spreading:} The normalized mass flux variations, measured at $\mathrm{x} / \mathrm{D}=$ 14 , as a function of $M_{J}$ for the circular jet are shown in Fig. 14. The corresponding screech stages (Fig. 11) are reproduced on the top for comparison. One finds that the fluxes undergo large variations with $\mathbf{M}_{\mathrm{J}}$. The fluxes are highest in the flapping mode $\mathrm{B}$, and are again relatively high in the next flapping mode $D$. With the onset of the helical mode $\mathrm{C}$, the flux values drop substantially. Pitot probe data, obtained at a single point on the jet centerline, reported in Refs. 31 and 32 are in general agreement with these results. For example, the total pressure was observed in those studies to be lower at pressure ratios corresponding to the $\mathrm{B}$ mode indicating an increased jet spreading.

Referring back to Fig. 7, it is reasonable to infer that without screech the fluxes for the circular jet would have been lower than those for the lobed nozzle. The occurrence of screech generally results in an increased jet spreading and this effect is most pronounced in the flapping modes. Note that the data in Fig. 7 for the circular jet $\left(\right.$ at $\left.\mathrm{M}_{\mathrm{J}}=1.63\right)$ involved screech in the $\mathrm{C}$ mode which is characterized by relatively less spreading. Therefore, the difference between the circular and the lobed nozzle data would have been even more dramatic at either a lower or a higher $M_{J}$ yielding the $B$ or $D$ screech modes for the circular nozzle.

Corresponding mass flux data, measured at $x / D$ $=14$, for all six cases are compared in Fig. 15. The fluxes for the elliptic and the rectangular jets are clearly high in the B' stage. The pattern is similar to the circular jet $B$ stage. However, it is not clear why the flux values 


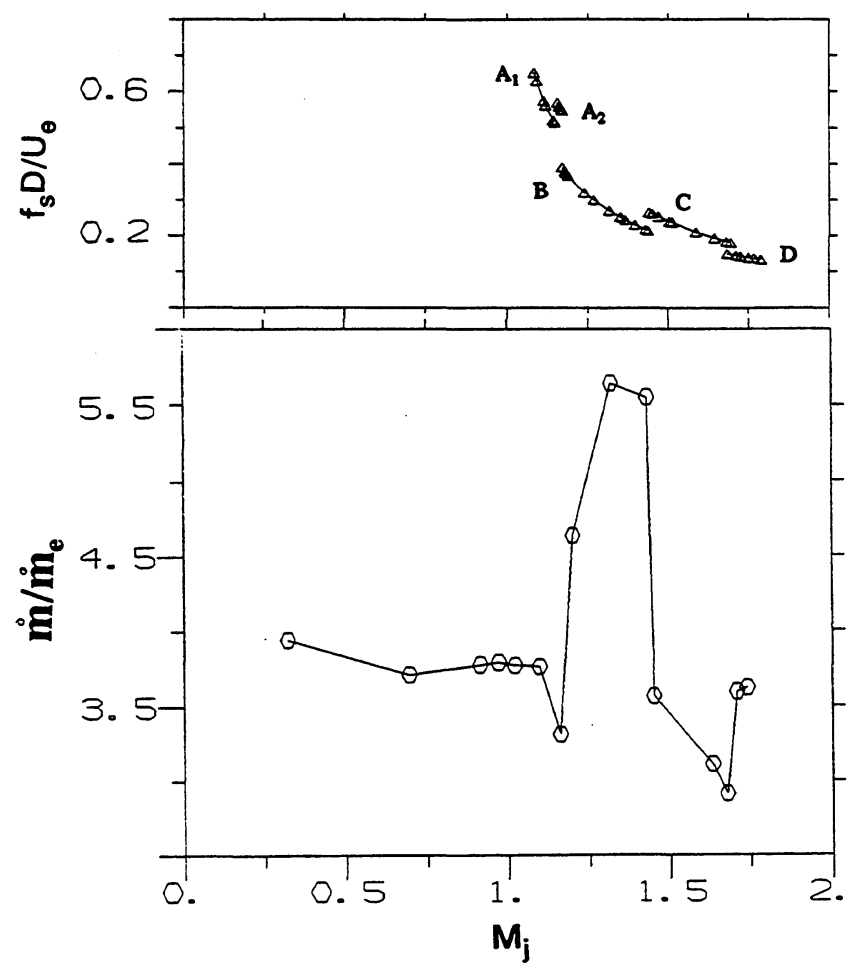

Fig. 14 Normalized mass flux measured at $x / D=14$, as a function of $\mathbf{M}_{\mathrm{j}}$, for the circular nozzle. Nondimensional screech frequency (Fig. 11) is shown on the top.

drop as the D' mode, which is also a flapping mode, is approached. It is possible that the screech amplitude, which diminishes as a stage jump is approached, is responsible for the trend. In general, however, it is clear that the flapping mode screech results in the highest jet spreading. This is consistent with the acoustic excitation results of Ref. 39 in which a combination of +1 and -1 modes (opposite sign helices) also yielded a remarkable jet spreading.

Referring back to the discussion earlier in this section, an additional reason for the relatively higher fluxes for the asymmetric jets, seen in Fig. 7, becomes clear. At $M_{\mathrm{j}}=1.63$, represented in Fig. 7, the elliptic and the rectangular jets went through a flapping mode screech whereas the circular jet went through a helical mode screech. This contributed to the observed higher fluxes for the asymmetric jets.

Once again, let us note that the fluxes for the tab cases in Fig. 15 are much larger throughout the Mach number range. The values are larger than those obtained with any of the asymmetric nozzles. This is true for supersonic as well as subsonic conditions. Note that

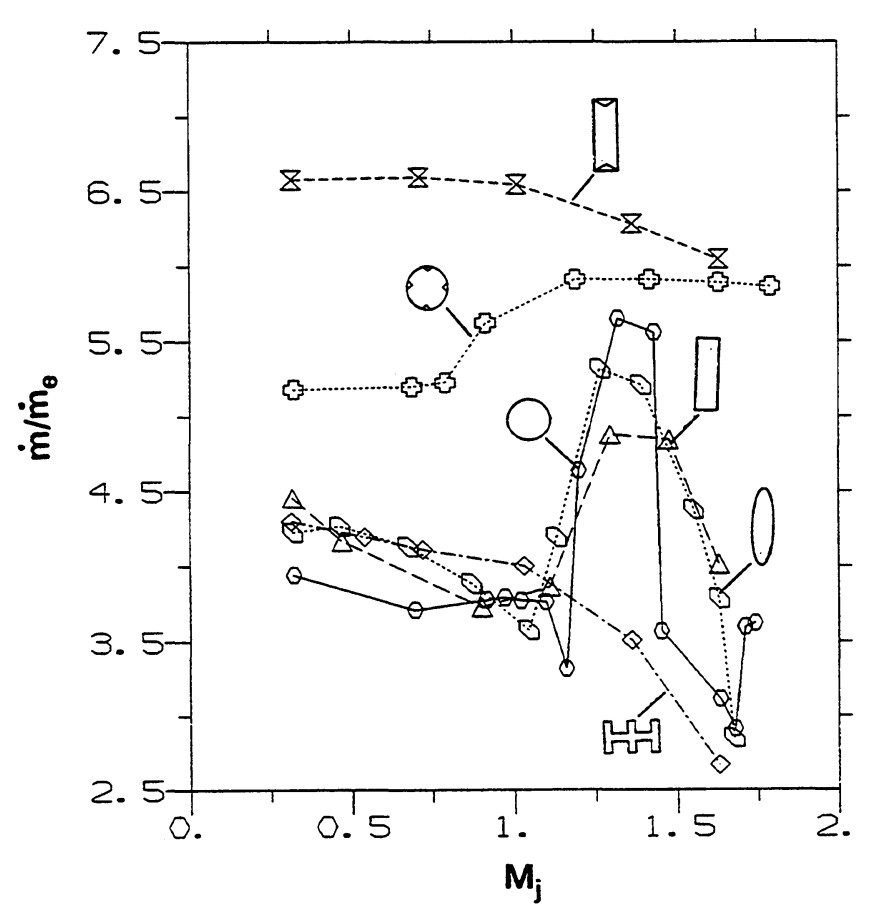

Fig. 15 Normalized mass flux measured at $x / D=14$, as a function of $\mathbf{M}_{\mathrm{J}}$, for all six cases.

especially the circular jet with tabs does not involve screech. Whereas the spreading increase with screech occurs through an organization of the azimuthal vortical structures and their subsequent dynamics, that with the tabs occurs primarily through the dynamics of the streamwise vortex pairs.

\subsection{Thrust loss for the tab cases:}

The measured thrust as a function of the nozzle pressure ratio is shown in Fig. 16 for the six cases. The data were obtained by a simple device involving a 'load cell' and an auxiliary plenum mounted on linear bearings, details of which can be found in Ref. 12. The data are shown in two sets, the lower set is for the circular jet and the upper set is for the other cases. The upper set is shifted by one major ordinate division. Since the circular jet had a smaller diameter, the corresponding thrust values are lower. In each set the ideal thrust is shown by the solid line. It becomes apparent that all the no tab cases involve slightly lower thrusts relative to the ideal value. The somewhat lower values are due to the boundary layers within the nozzles. The ideal thrust is calculated with the assumption of uniform flow with zero boundary layer thickness. 


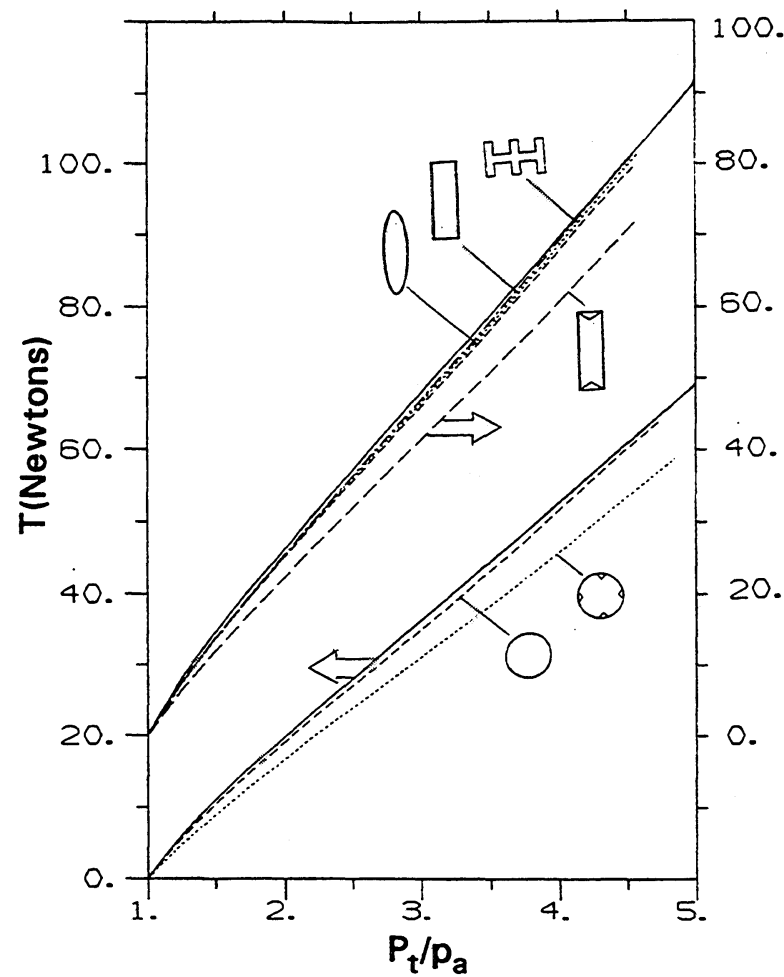

Fig. 16 Thrust versus nozwle pressure ratio for the six cases; two solid curves represent isentropic predictions.

When the tabs are added, as expected, there is considerable thrust loss. For example, at $\mathrm{M}_{\mathrm{J}}=1.63$ $\left(\mathrm{P}_{\mathrm{T}} / \mathrm{p}_{\mathrm{A}}=4.47\right)$, the thrust loss for either of the two tab cases in Fig. 16 was approximately $12 \%$. However, with the tabs in place, there is area blockage and the mass flow is lower for a given $P_{T} / p_{A}$. An appropriate evaluation of the performance of a given nozzle configuration is obtained by measurement of the thrust coefficient, which, for the six cases, is shown in Fig. 17. These data required simultaneous measurement of the actual mass flow which was done by an orifice meter located in the supply line. Unfortunately, the uncertainty in the mass flow data was relatively large and resulted in a scatter of about $\pm 2 \%$ in the thrust coefficient.

It should also be mentioned here that the ideal thrust, used to obtain $C_{f}$, was calculated by assuming convergent flow (which was the case). For a given $P_{T} / p_{A}$, full expansion (via convergent-divergent nozzle) would yield the maximum available thrust. ${ }^{10}$ This would be higher, for example, by about $2 \%$ at $\mathrm{P}_{\mathrm{T}} / \mathrm{p}_{\mathrm{A}}=4.47$. If this were used in the calculation, the thrust coefficients would have correspondingly lower values. Note also that the data

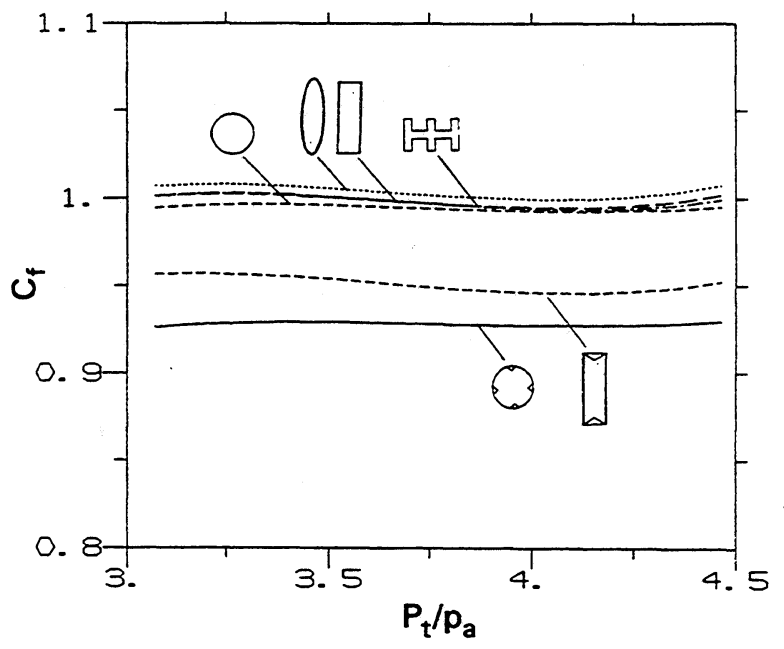

Fig. 17 Thrust coefficient versus nozzle pressure ratio for the six cases.

in Fig. 17 are shown only in a high range of $P_{T} / p_{A}$ where the measurement uncertainty was lower and within the limits stated in the foregoing. Least-squares-fitted curves through approximately six data points are shown.

As expected, the thrust coefficient turns out to be nearly unity for all the no-tab cases throughout the nozzle pressure ratio range covered in Fig. 17. With the tabs, of course, there is a performance penalty. However, the penalty is only about $5 \%$ for the rectangular jet with two tabs, and about $7 \%$ for the circular jet with four tabs. Recall that the corresponding geometric area blockages were about $12 \%$ and $8 \%$, respectively. Thus, the percentage penalty for the circular jet case turns out to be about the same as the area blockage. The corresponding penalty for the rectangular case, on the other hand, is lower. The difference borders on the uncertainty in the data but appears to be real. Unfortunately, not much can be said about the fluid dynamics from the thrust data, and an explanation for the difference will require further investigation of the flow field close to the tabs. It should be pointed out that in the case of four tabs, as opposed to two, the flow has to negotiate more corners. Also, with the circular nozzle, fabrication resulted in a small part of the tab, (sector determined by the base of the tab and the curved nozzle wall), standing perpendicular to the flow. In the rectangular nozzle case, the entire tab surface is at $45^{\circ}$ to the stream direction. These factors could explain the relatively higher performance penalty in the tab case with the circular nozzle. 


\section{Concluding Remarks:}

The spreading characteristics of free jets from various asymmetric nozzles have been studied. The nozzles included a circular, a 3:1 rectangular, a 3:1 elliptic and a six-lobed one. In addition, jets from the rectangular nozzle fitted with two tabs on the narrow edges and the circular nozzle fitted with four equally spaced tabs were also included in the comparative study. Measurements covered incompressible subsonic conditions to underexpanded supersonic conditions up to a jet Mach number of 1.96 .

Measurements in the asymptotic regions of the circular jet show that the entrainment rate, when normalized by the initial mass flux, the nozzle diameter and the ratio of the density between the ambient and the nozzle exit, is a constant. The constant has a value of approximately 0.27 , agreeing with previous results for incompressible jets. Most asymmetric jets, however, did not reach the asymptotic state within the measurement range covering thirty equivalent diameters from the nozzle exit. Screech, nozzle shape and the tabs altered the jet spreading within the developing regions very significantly.

At subsonic conditions the spreading of the asymmetric jets was found to be only slightly more than that of the circular jet. But it was found to be significantly more at supersonic conditions when screech occurred. Screech increased the jet spreading with all nozzles, the amount of increase varying with varying stages of the screech. The lobed nozzle which did not involve screech, therefore, exhibited the least spreading at supersonic conditions.

The unsteady flow characteristics under the various stages of screech, for the circular and the rectangular nozzle have been studied via phase averaged measurement of the fluctuating total pressure. The axisymmetric, flapping and helical unsteady motions occurring in the vaious stages of screech with the circular nozzle are vividly demonstrated by these data. Two stages of screech are observed with the rectangular and the elliptic nozzles. Both stages involve the flapping unsteady motion. Among the various stages, screech involving the flapping motion caused the maximum jet spreading.

Jet spreading for the two tab configurations was found to be the largest among all the cases. This was true in the subsonic regime, as well as in the supersonic regime in spite of the fact that screech was eliminated by the tabs. The dynamics of the streamwise vortex pairs produced by the tabs caused the most efficient jet spreading. Thus, a manipulation of the latter dynamics is likely to hold the key to possible further increases in jet spreading needed in various applications.

\section{Acknowledgement:}

The author wishes to thank Dr. Judith Foss for help with some of the experiments, to her and Dr. Milo Dahl for helpful discussions, to Drs. Ganesh Raman and Jay Panda for reviewing the manuscript, and to Mr. Tom Norum of NASA LaRC for helpful inputs on the aspects of screech.

\section{References:}

${ }^{1}$ Ho C.-M. \& Gutmark E., "Vortex induction and mass entrainment in a small-aspect-ratio elliptic jet", J. Fluid Mech., vol. 179, pp. 383-405, 1987.

2 Hussain F. \& Husain H.S., "Elliptic jets. Part 1. Characteristics of unexcited and excited jets", J. Fluid Mech., 208, pp. 257-320 1989.

${ }^{3}$ Trestacoste, N. \& Sforza, P., "Further experimental results for three-dimensional free jets", ALAA J., 5(5), May, 1967.

'Krothapalli, A., Baganoff, D. \& Karamcheti, K., "On the mixing of rectangular jet", J. Fluid Mech., 107, p.201, 1981.

${ }^{5}$ Tsuchiya, Y., Horikoshi, C. \& Sato, T., "On the spread of reactangular jets", Experiments in Fluids, 4, p. 197, 1986.

${ }^{6}$ Morrison, G.L., Swan, D.H. \& DeOtte, R.E., "Development of the mean velocity distribution in rectangular jets", ALAA Paper 92-0505, 1992.

${ }^{7}$ Grinstein, F.F., "Vorticity dynamics in spatially-developing rectangular jets", ALAA Shear Flow Conf. (933286), Orlando, FL, July 1993.

schadow K.C., Wilson K.J., Lee M.J. \& Gutmark E., "Enhancement of mixing in reacting fuel-rich plumes issued from elliptic nozzles", J. Propulsion, 3, p. 145, 1987.

'Seiner, J. M. \& Krejsa, E.A., "Supersonic jet noise and high speed civil transport", ALAA Paper 89-2358, 1989.

- Papamoschou, D. \& Roshko, A., "The compressible turbulent shear layer: an experimental study", J. Fluid Mech., 197, 453-477, 1988.

1 Ahuja K. K. \& Brown W. H., "Shear flow control by mechanical tabs", ALAA Paper No. 89-0994, (1989).

12 Zaman K.B.M.Q., Reeder M.F., \& Samimy M., "Control of an axisymmetric jet using vortex generators", Physics of Fluids A, 6 (2), pp. 778-793, Feb., 1993. 
${ }^{13}$ Surks, P., Rogers, C.B. \& Parekh, D.E., "Entrainment and acoustic variations in a round jet from introduced streamwise vorticity", ALAA J., 32(10), 2108-2110, 1994

${ }^{14}$ Zaman, K.B.M.Q., "Axis switching and spreading of an asymmetruc jet - role of vorticity dynamics", ALAA Paper 95-0889, 1995.

is Bohl, D. \& Foss, J., "Enhancement of passive mixing tabs by the addition of secondary tabs", ALAA Paper 960545, 1996.

16 Zhang, S. \& Schneider, S.P., "Molecular-mixing measurements and turbulent-structure visualizations in a round jet with tabs", ALAA Paper No. 94-3082, 1994.

17 King, C.J., Krothapalli, A. and Strykowski, P.J., "Streamwise vorticity generation in supersonic jets with minimal thrust loss", ALAA Paper No. 94-0661, 1994.

${ }^{18}$ Kobayashi, H., Oinuma, H., Sawamura, T. and Outa, E., "Effect of tab size on supersonic underexpanded cold and heated jet noise suppression and jet thrust loss", ALAA Paper No. 93-4348, 1993.

${ }^{19}$ Crow S.C. \& Champagne F.H., "Orderly structures in jet turbulence", J. Fluid Mech., 48, p.547, 1971.

20 Abramovich, G.N., "The theory of turbulent jets", The M.I.T. Press, 1963.

21 Zaman K.B.M.Q., "Flow Field and Near and Far Sound Field of a Subsonic Jet", J. Sound \& Vib., 106, 1-16, 1986.

2 Wygnanski, I. \& Fiedler, H., "Some measurements in the self-preserving jet", J. Fluid Mech., 38, p.577, 1969.

${ }^{2}$ Ricou, F.P. \& Spalding, D.B., "Measurements of entrainment by axisymmetrical turbulent jets", J. Fluid Mech., 11, 21-32, 1961.

${ }^{2}$ Witze, P.O., "Centerline velocity decay of compressible free jets", ALAA J., 12(4), p.417, April, 1974.

${ }^{5}$ Liepmann, D. \& Gharib, M., "The role of streamwise vorticity in the near-field entrainment of round jets", $J$. Fluid Mech., 245, 643-668, 1992.

${ }^{26}$ Powell, A., "On the mechanism of choked jet noise", Proc. Physical Soc. (London), B66, p. 1039, 1953.

${ }^{27}$ Davis, M.G. \& Oldfield, D.E.S., "Tones from a choked axisymmetric jet. I. Cell structure, eddy velocity and source locations", Acustica, vol. 12, no 4, pp. 257 $277,1962$.

${ }^{2}$ Norum, T.D., "Screech suppression in supersonic jets", ALAA J., 21, pp. 235-240 (1983).

29 Seiner, J.M., "Advances in high speed jet aeroacoustics", ALAA Paper 84-2275, 1984.

${ }^{30}$ Tam, C.K.W., "Jet noise generated by large-scale coherent motion", NASA RP 1258, vol 1, Aug., 1991.

31 Glass, D.R., "Effects of acoustic feedback on the spread and decay of supersonic jets", ALAA J., $\sigma(10)$, 1890-1897, 1968.
32 Sherman, P.M., Glass, D.R. \& Duleep, K.G., "Jet flow field during screech", Applied Sci. Res., 32(3), 283303, 1976.

${ }^{33}$ Krothapalli, A., Hsia, Y., Baganoff, D. \& Karamcheti, $\mathrm{K}$., "The role of screech tones in mixing of an underexpanded rectangular jet", J. Sound \& Vib., 106(1), 119143, 1986.

${ }^{34}$ Raman, G., Rice, E.J. \& Reshotko, E., "Control of an axisymmetric turbulent jet by multi-modal excitation", Proc. 8th Turb. Sh. Flow Conf., 6-2, Technical University of Munich, Sept., 1991.

${ }^{35}$ Powell, A., Umeda, Y. \& Ishii, R., "The screech of round choked jets, revisited", ALAA Paper 90-3980, 1990.

36 Panda, J., "Measurement of shock oscillation in underexpanded supersonic jets", ALAA Paper 95-2145, 1995.

${ }^{37}$ Ponton, M.K. \& Seiner, J.M., "Acoustic study of B helical mode for choked axysymmetric nozzle", ALAA J., 33(3), 413-420, 1995.

3 Raman, G. \& Rice, E.J., "Supersonic jet mixing enhancement using impingement tones from obstacles of various geometries", ALAA J., 33(3), 454-462, 1995.

39 Parekh, D.E., Reynolds, W.C. \& Mungal, M.G., 1987, "Bifurcation of round air jets by dual-mode acoustic excitation", ALAA Paper No. 87-0164.

- Shapiro, A.H., "The dynamics and thermodynamics of compressible fluid flow", The Ronald Press Co., New York, 1953. 
Public reporting burden for this collection of information is estimated to average 1 hour per response, including the time for reviewing instructions, searching existing data sources, gathering and maintaining the data needed, and completing and reviewing the collection of information. Send comments regarding this burden estimate or any other aspect of this collection of information, including suggestions for reducing this burden, to Washington Headquarters Services, Directorate for Information Operations and Reports. 1215 Jefferson Davis Highway, Suite 1204, Arlington, VA 22202-4302, and to the Office of Management and Budget, Papenwork Reduction Project (0704-0188). Washington. DC 20503.

\begin{tabular}{|l|l|l} 
1. AGENCY USE ONLY (Leave blank) & $\begin{array}{c}\text { 2. REPORT DATE } \\
\text { December } 1995\end{array}$ & $\begin{array}{c}\text { 3. REPORT TYPE AND DATES COVERED } \\
\text { Technical Memorandum }\end{array}$
\end{tabular}

4. TITLE AND SUBTITLE

Spreading Characteristics and Thrust of Jets From Asymmetric Nozzles

6. AUTHOR(S)

K.B.M.Q. Zaman

7. PERFORMING ORGANIZATION NAME(S) AND ADDRESS(ES)

National Aeronautics and Space Administration

Lewis Research Center

Cleveland, Ohio 44135-3191

9. SPONSORING/MONITORING AGENCY NAME(S) AND ADDRESS(ES)

National Aeronautics and Space Administration

Washington, D.C. 20546-0001

5. FUNDING NUMBERS

WU-537-02-22

8. PERFORMING ORGANIZATION

REPORT NUMBER

$E-10062$

10. SPONSORING/MONITORING AGENCY REPORT NUMBER

NASA TM-107132

AIAA-96-0200

11. SUPPLEMENTARY NOTES

Prepared for the 34th Aerospace Sciences Meeting and Exhibit sponsored by the American Institute of Aeronautics and Astronautics, Reno, Nevada, January 15-18, 1996. Responsible person, K.B.M.Q. Zaman, organization code 2660, (216) 433-5888.

12a. DISTRIBUTIONAVAILABILTTY STATEMENT

12b. DISTRIBUTION CODE

Unclassified - Unlimited

Subject Category 02

This publication is available from the NASA Center for Aerospace Information, (301) 621-0390.

13. ABSTRACT (Maximum 200 words)

The spreading characteristics of jets from several asymmetric nozzles are studied in comparison to those of an axisymmetric jet, over the Mach number $\left(\mathrm{M}_{\mathrm{J}}\right)$ range of 0.3 to 1.96. The effect of tabs in two cases, the axisymmetric nozzle fitted with four tabs and a rectangular nozzle fitted with two large tabs, is also included in the comparison. Compared to the axisymmetric jet, the asymmetric jets spread only slightly faster at subsonic conditions, while at supersonic conditions, when screech occurs, they spread much faster. Screech profoundly increases the spreading of all jets. The effect varies in the different stages of screech, and the corresponding unsteady flowfield characteristics are documented via phaseaveraged measurement of the fluctuating total pressure. An organization and intensification of the azimuthal vortical structures under the screeching condition is believed to be responsible for the increased spreading. Curiously, the jet from a 'lobed mixer' nozzle spreads much less at supersonic conditions compared to all other cases. This is due to the absence of screech with this nozzle. Jet spreading for the two tab configurations, on the other hand, is significantly more than any of the no-tab cases. This is true in the subsonic regime, as well as in the supersonic regime in spite of the fact that screech is essentially eliminated by the tabs. The dynamics of the streamwise vortex pairs produced by the tabs cause the most efficient jet spreading thus far observed in the study.

\section{SUBJECT TERMS}

Jets; Mixing; Mixing layers; Tabs; Thrust

$\mathrm{A} 03$

\begin{tabular}{|c|c|c|}
\hline $\begin{array}{c}\text { 17. SECURTY CLASSIFICATION } \\
\text { OF REPORT } \\
\text { Unclassified }\end{array}$ & $\begin{array}{c}\text { 18. SECURITY CLASSIFICATION } \\
\text { OF THIS PAGE } \\
\text { Unclassified }\end{array}$ & $\begin{array}{c}\text { 19. SECURITY CLASSIFICATION } \\
\text { OF ABSTRACT } \\
\text { Unclassified }\end{array}$ \\
\hline
\end{tabular}

